\title{
OPEN In situ-forming collagen hydrogel crosslinked via multi-functional PEG as a matrix therapy for corneal defects
}

\author{
Gabriella Maria Fernandes-Cunha ${ }^{1}$, Karen Mei Chen ${ }^{1}$, Fang Chen ${ }^{1}$, Peter Le ${ }^{1}$, Ju Hee Han ${ }^{1}$, \\ Leela Ann Mahajan ${ }^{1}$, Hyun Jong Lee ${ }^{2}$, Kyung Sun $\mathrm{Na}^{3}$ \& David Myung ${ }^{1,4,5 \bowtie}$
}

Visually significant corneal injuries and subsequent scarring collectively represent a major global human health challenge, affecting millions of people worldwide. Unfortunately, less than $2 \%$ of patients who could benefit from a sight-restoring corneal transplant have access to cadaveric donor corneal tissue. Thus, there is a critical need for new ways to repair corneal defects that drive proper epithelialization and stromal remodeling of the wounded area without the need for cadeveric donor corneas. Emerging therapies to replace the need for donor corneas include pre-formed biosynthetic buttons and in situ-forming matrices that strive to achieve the transparency, biocompatibility, patient comfort, and biointegration that is possible with native tissue. Herein, we report on the development of an in situ-forming hydrogel of collagen type I crosslinked via multi-functional polyethylene glycol (PEG)-N-hydroxysuccinimide (NHS) and characterize its biophysical properties and regenerative capacity both in vitro and in vivo. The hydrogels form under ambient conditions within minutes upon mixing without the need for an external catalyst or trigger such as light or heat, and their transparency, degradability, and stiffness are modulated as a function of number of PEG arms and concentration of PEG. In addition, in situ-forming PEG-collagen hydrogels support the migration and proliferation of corneal epithelial and stromal cells on their surface. In vivo studies in which the hydrogels were formed in situ over stromal keratectomy wounds without sutures showed that they supported multi-layered surface epithelialization. Overall, the in situ forming PEG-collagen hydrogels exhibited physical and biological properties desirable for a corneal stromal defect wound repair matrix that could be applied without the need for sutures or an external trigger such as a catalyst or light energy.

Injuries and diseases of the cornea that lead to stromal defects are significant causes of disability, morbidity and vision loss worldwide ${ }^{1,2}$. In cases of corneal thinning or melt situations where perforation is imminent, off-label use of cyanoacrylate is employed to stabilize the cornea prior to a definitive but invasive corneal transplant ${ }^{3}$. However, cyanoacrylate glue creates an opaque, rough surface that provides no visual or regenerative benefits ${ }^{4}$. Penetrating and lamellar keratoplasty are invasive procedures and the waiting lists for human corneal transplantation include more than 10 million people globally ${ }^{5}$. Numerous efforts to create pre-formed biosynthetic donor corneal buttons using collagen, gelatin, and hyaluronic acid have been made ${ }^{2,6-10}$. Recently, Holoclar, a fibrinbased disc with stem cells was approved for use throughout the European Union to restore vision in patients with physical injuries and chemical burns ${ }^{11}$.

Recently, matrix therapies of various kinds have been developed ${ }^{2-4,8,12,13}$ where a flowable material is applied and cured over corneal wounds. The goal of a matrix therapy is to sustain epithelial and stromal cell ingrowth ${ }^{4}$, and specific priorities are transparency and biocompatibility to improve host-graft interaction ${ }^{6,14}$. In one study, dopamine hydrazone-crosslinked hyaluronic acid hydrogels were developed to enhance adhesivity to the corneal tissue and deliver limbal cells ${ }^{8}$. Samarawickrama et al. showed that collagen-like peptide conjugated to

\footnotetext{
${ }^{1}$ Ophthalmology, Byers Eye Institute, Stanford University School of Medicine, Palo Alto, CA, USA. ${ }^{2}$ Chemical and Biological Engineering, Gachon University, Seongnam-si, Gyeonggi-do, Republic of Korea. ${ }^{3}$ Ophthalmology and Visual Science, Yeouido St. Mary's Hospital, College of Medicine, The Catholic University of Korea, Seoul, Republic of Korea. ${ }^{4}$ Chemical Engineering, Stanford University, Stanford, CA, USA. ${ }^{5}$ VA Palo Alto HealthCare System, Palo Alto, CA, USA. ${ }^{\boxplus}$ email: djmyung@stanford.edu
} 
1

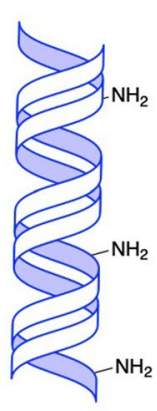

2

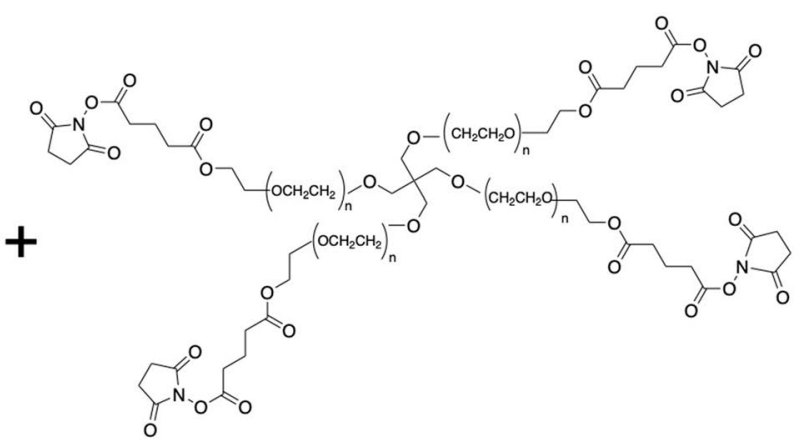

3

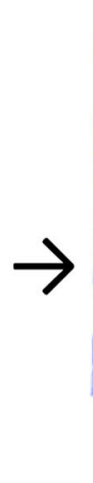

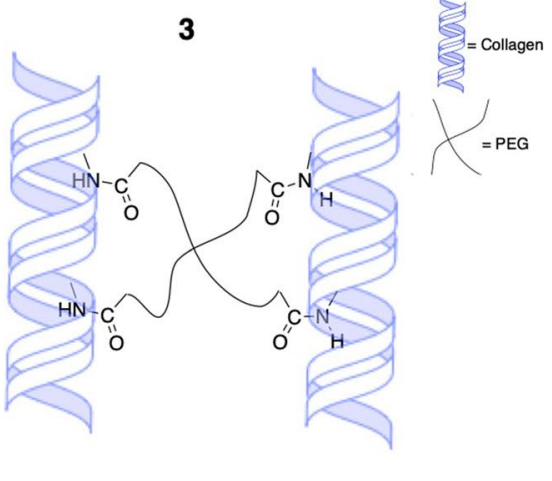

Figure 1. Schematic of the crosslinking between primary amines on collagen and NHS groups on multi-arm PEG polymers; (1) collagen, (2) 4-arm PEG-NHS (3) PEG-collagen hydrogel.

PEG-maleimide was created to seal acute corneal perforations while promoting tissue regeneration ${ }^{3}$. Collagen is a particularly attractive biomaterial for ocular adhesives because it is the most abundant protein in mammals, is naturally present in corneal stroma and is the main structural protein in the extracellular environment ${ }^{15}$. However, for collagen to be used in situ on a wounded corneal surface, the cytotoxicity of the chemistry being used to crosslink is a critical consideration $3,9,12,16,17$.

Recently, both photo-initiated and light-free in situ-forming hydrogel approaches have been shown to have promise in the repair of corneal stromal defects. Sani et al. demonstrated that a photocrosslinked methacryloylgelatin gel exhibited adhesive properties and supported surface epithelialization ${ }^{18}$. Li et al. reported on a similar photocurable gelatin-based method where acrylated gelatin is reacted with thiolated gelatin ${ }^{13}$. While these strategies do not require sutures, they do necessitate a photoinitiator and light energy for gelation. Although photochemistry has certain potential advantages and is used clinically and is FDA-approved for corneal crosslinking (CXL) via riboflavin, it also has known detrimental effects on stromal keratocytes and corneal nerves ${ }^{19}$. We recently reported on an in situ-forming collagen hydrogel that is crosslinked by bio-orthogonal copper-free click chemistry without the need for external triggers such as light, heat, or any chemical initiators, and demonstrated that it can support a multi-layered epithelium in corneal organ culture ${ }^{12}$.

Here, we present a light-free, in situ-forming approach to crosslinking collagen using multi-arm PEG using $\mathrm{N}$-hydroxysuccinimide (NHS) chemistry to fill and repair corneal defects. This chemical crosslinking approach has been used clinically in sealants to stop fluid leaks in both neurological and ophthalmic surgery ${ }^{20}$. For instance, the FDA-approved product ReSure (Ocular Therapeutix, Bedford, MA) is a sealant for corneal incisions which utilizes an (NHS)-terminated 4-arm PEG prepolymer that reacts with the primary amines of trilysine to facilitate in situ gelation ${ }^{21}$. In other work, collagen has been crosslinked via multifunctional PEG to form gels that have been shown to be biocompatible and support cell growth in vitro ${ }^{17}$. Given its track record as a crosslinking chemistry for an ophthalmic sealant for corneal incisions ${ }^{4}$, we have utilized the succinimide active ester reaction to facilitate crosslinking of collagen in situ as a matrix therapy for corneal epithelial and stromal defect repair. The experiments reported herein were used to investigate the importance of PEG arm number and concentration on the material's biophysical properties both in vitro and in vivo, and demonstrate the potential of multi-arm PEG-collagen hydrogels as in situ-forming, suture-free, and catalyst-free scaffolds to promote epithelial wound healing in the treatment of corneal defects.

\section{Results}

Mechanical properties of PEG-collagen hydrogels. Here we applied NHS ester chemistry to crosslink varying concentrations of collagen using multi-arm PEG linkers in situ to generate hydrogels that can fill corneal defects and promote re-epithelialization (Fig. 1). The collagen hydrogels were crosslinked with various concentrations (4, 8 and 16\% v/v PEG to collagen) of 4-arm and 8-arm PEG-NHS resulting in distinct hydrogels with different mechanical properties depending on the PEG concentration and arm number. The mechanical properties of PEG-collagen hydrogels were measured using rheological methods (Fig. 2a,b,c). The non-crosslinked collagen hydrogel reached $\approx 85 \mathrm{~Pa}$ in $300 \mathrm{~s}$ and this value increased to $\approx 141 \mathrm{~Pa}$ until $900 \mathrm{~s}$. For the PEG-collagen hydrogel, the storage modulus was modulated by the PEG crosslinker concentration and by the PEG arm number. The storage modulus of 4\% 4-arm PEG-collagen hydrogel steadily increased to $\approx 1000 \mathrm{~Pa}$ through $900 \mathrm{~s}$. The storage modulus of 4-arm PEG-collagen with $8 \%$ PEG content was similar to that with $4 \%$, while the 4-arm PEG-collagen with $16 \%$ PEG content had a storage modulus that remained lower on the order of $\approx 500 \mathrm{~Pa}$. The storage moduli for the 8-arm PEG-collagen hydrogels with 4\% PEG were very similar to those of the 4-arm PEGcollagen hydrogels. However, the 8-arm PEG-collagen with 8\% PEG content reached a higher storage modulus compared to the 8 -arm PEG-collagen with $4 \%$ PEG; at $900 \mathrm{~s}$ the storage modulus was $\approx 1320 \mathrm{~Pa}$. The Fig. $2 \mathrm{~b}$ shows that there was no statistical difference in the storage modulus of collagen crosslinked with 4 arm PEGcollagen at concentrations of 4 and $8 \%$. Collagen crosslinked with both 4 -arm and 8 -arm PEG using high PEG concentration $(16 \%)$ had storage moduli that were significantly smaller $\left({ }^{* * *} \mathrm{p}<0.0001\right)$ compared to those of 4 and 8 arm PEG-collagen with 4 and $8 \%$ PEG concentrations. Collagen crosslinked with both 4 -arm and 8-arm 
a

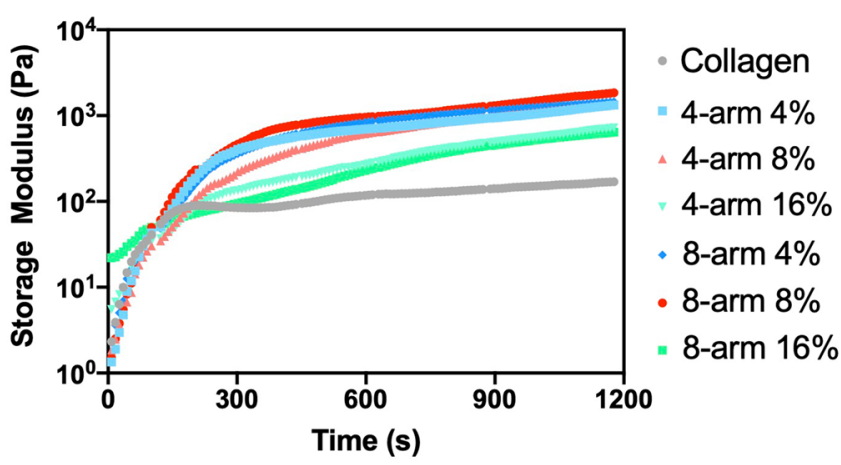

C

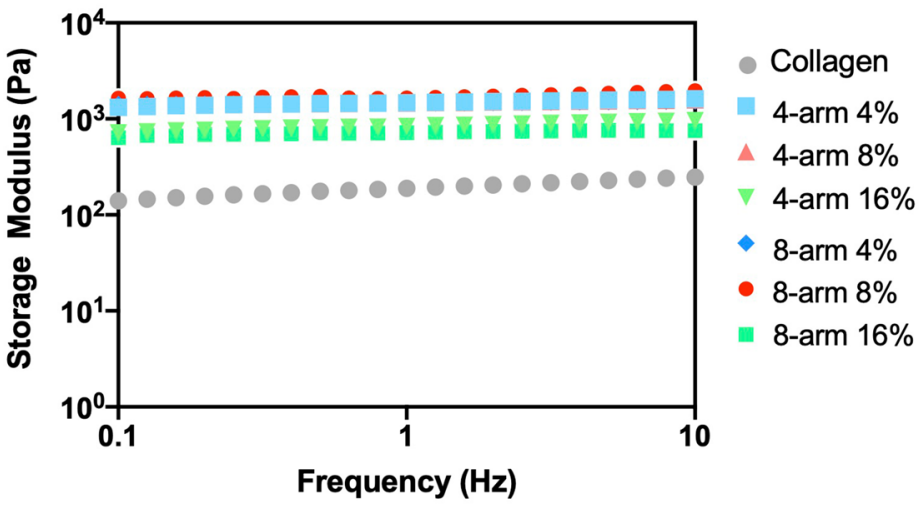

b

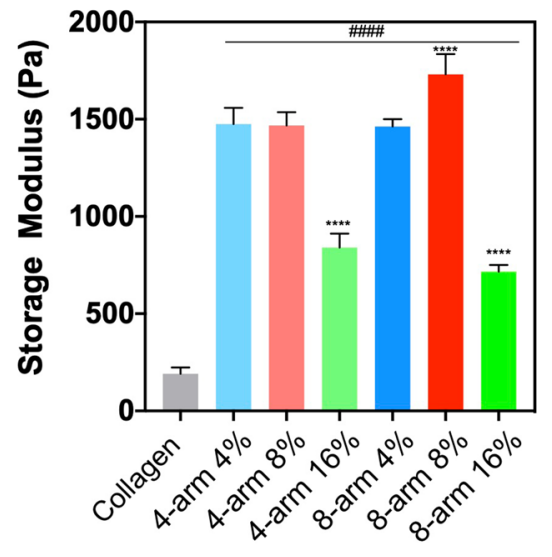

d

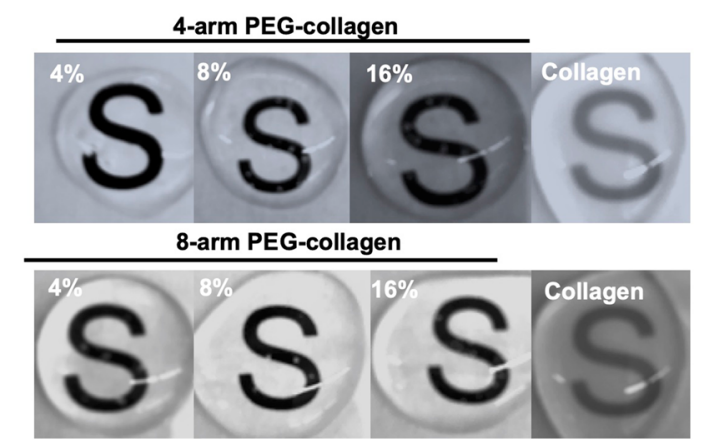

Figure 2. Physical properties of chemically crosslinked PEG-collagen hydrogels by NHS chemistry and noncrosslinked (physical) collagen hydrogels. (a) Dynamic moduli of 4-arm PEG-collagen and 8-arm PEG-collagen hydrogels using different concentrations of PEG polymer as function of time during gelation. The hydrogels were mounted immediately after mixing. (b) Dynamic moduli of 4-arm PEG-collagen and 8-arm PEG-collagen hydrogels at different concentrations of PEG polymer. 4 and 8-arm PEG-collagen at 16\% v/v PEG show lower storage modulus $\left({ }^{* * *} \mathrm{p}<0.0001\right)$ compared to 4 and 8arm PEG-collagen at 4 and $8 \%$. 8-arm PEG-collagen hydrogel at $8 \%$ showed higher storage modulus $\left({ }^{* * *} \mathrm{p}<0.0001\right)$ compared to all other PEG concentrations and type. Collagen crosslinked with 4 and 8 -arm PEG polymer at all concentrations showed significant higher storage modulus (\#\#\# $\mathrm{p}<0.0001$ ) compared to non-crosslinked collagen hydrogel; data is presented as mean \pm SD, two-way ANOVA ( $\mathrm{p}<0.005$ ) was used to detect statistical differences followed by Tukey's multiple comparisons test. (c) Dynamic moduli of 4-arm PEG-collagen and 8-arm PEG-collagen hydrogels as a function of frequency. (d) Photographs of 4 and 8-arm PEG-collagen and non-crosslinked (physical) collagen hydrogels.

PEG at all concentrations had a higher storage modulus compared to non-crosslinked collagen (\#\#\# $<0.0001$ ). The highest storage moduli were achieved by using 8 -arm PEG at $8 \% \mathrm{v} / \mathrm{v}$ compared to all the other groups $\left({ }^{* * * *} \mathrm{p}<0.001\right)$. Thus, higher number of PEG arms increases the modulus at higher PEG concentration (up to $8 \%)$. The storage moduli of all the hydrogels at $1200 \mathrm{~s}$ is summarized in supplementary Table 1 and represents the moduli of the hydrogels used in the in vitro experiments. To confirm that gelation was completed, the hydrogels' storage and loss moduli were measured as a function of frequency from 0.1 to $10 \mathrm{~Hz}$ (Fig. 2c,d). The moduli did not vary as a function of the frequency.

The optical properties of the 4 and 8-arm PEG-collagen and non-crosslinked (physical) collagen hydrogels were analyzed to determine if the crosslinked hydrogels were suitable for use in the cornea. 4 and 8-arm PEG-collagen hydrogels with 4, 8 and 16\% PEG content were observed to be relatively transparent while the non-crosslinked collagen hydrogel was relatively opaque (Fig. 2d). To quantify this change in transparency, hydrogel transmittance was evaluated at wavelengths between 300 and $750 \mathrm{~nm}$, before and after hydrogel swelling (Fig. 3a,b). The transmittance of 4-arm and 8-arm PEG-collagen hydrogels (with 4 and $8 \%$ PEG content) remained constant at $\approx 80 \%$ in the visible light range. The transmittance of 4 -arm PEG-collagen with $16 \%$ PEG content increased with wavelength from $\approx 20 \%$ to $75 \%$, while for the 8-arm PEG-collagen with $16 \%$ PEG increased from $\approx 30 \%$ to $80 \%$. The non-crosslinked collagen hydrogel increased from $\approx 5 \%$ to $70 \%$. The transmittance after swelling remained similar to that before swelling; however, for the 8-arm PEG-collagen with 16\% PEG content the transparency decreased. While for other types of collagen hydrogels, swelling results in increase in transparency, this was not observed for the PEG-collagen hydrogels developed in this study ${ }^{9}$. In the presence of corneal cells the transmittance of the 4 and 8-arm PEG-collagen hydrogels with 16\% PEG content improved 
a

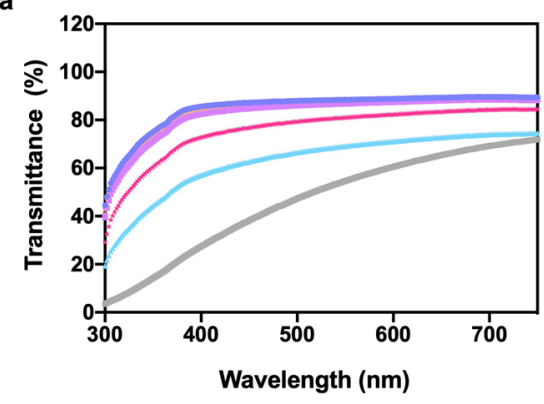

c

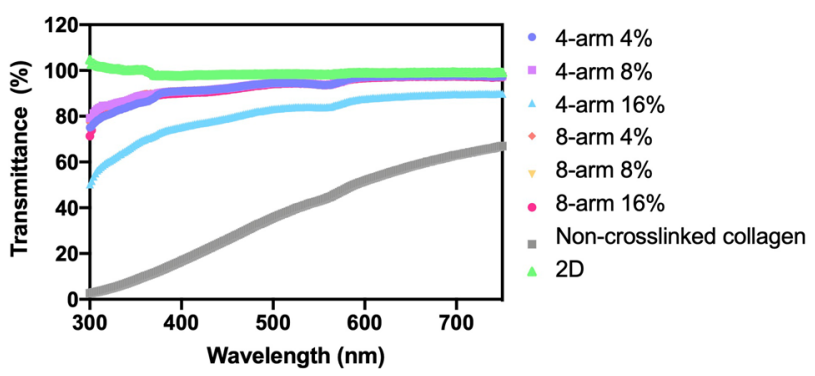

4-arm $4 \%$

4-arm $8 \%$

4-arm 16\%

8 -arm $4 \%$

8 -arm $8 \%$

8-arm $16 \%$

Non-crosslinked collagen b

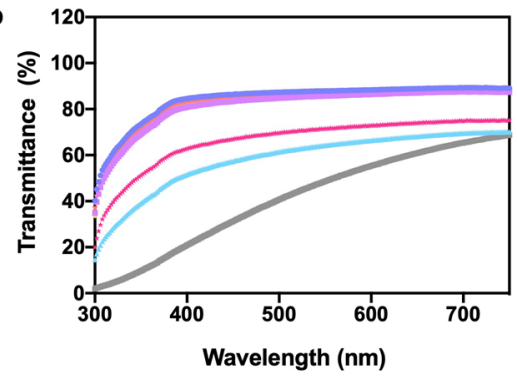

4-arm 4\%

4-arm $8 \%$

4-arm $16 \%$

8 -arm $4 \%$

8 -arm $8 \%$

8 -arm $16 \%$

- Non-crosslinked collagen

d

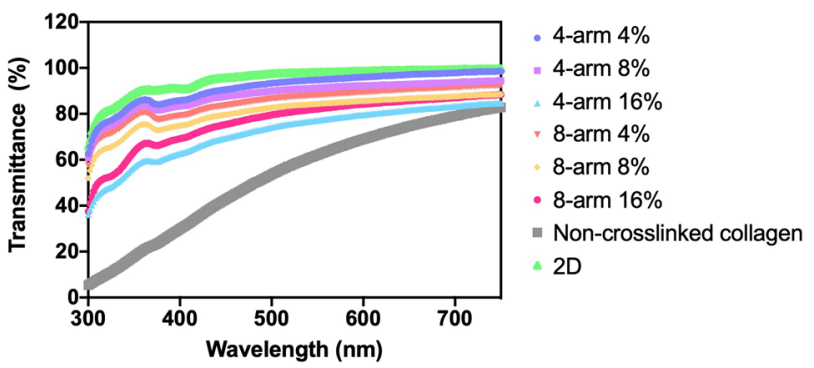

Figure 3. Transmittance spectra of collagen hydrogels from 350 to $800 \mathrm{~nm}$ (a) before swelling (b) after swelling for $24 \mathrm{~h}$ in PBS, (c) after $24 \mathrm{~h}$ in the presence of ICECs and (d) after $24 \mathrm{~h}$ in the presence CSSCs.

compared to the hydrogels at the same concentration without the cells, after swelling (Fig. 3c,d). In the presence of human immortalized corneal epithelial cells (ICEC), the transmittance of 4-arm PEG-collagen hydrogel at 16\% PEG increased from $\approx 50 \%$ to $90 \%$ and had light transmittance $\approx 80 \%$ at $500 \mathrm{~nm}$, while for 8 -arm PEG-collagen with $16 \%$ PEG, the light transmittance increased from $\approx 70 \%$ to $90 \%$. Similar improvement in the transmittance was observed for the PEG-collagen hydrogels in the presence of corneal stromal stem cells (CSSC). Transmittance for both 4 and 8-arm PEG-collagen hydrogel with 16\% PEG increased from $\approx 40 \%$ to $85 \%$ and showed a light transmittance of $\approx 75$ at $500 \mathrm{~nm}$. Bektas et al. observed that the transmittance of keratocyte-loaded 3D bioprinted GelMA hydrogels decreased compared to the hydrogels without cells, but then increased with prolonged time in culture $^{7}$. We believe that a decrease in light transmittance in the presence of cells was not observed in this study due to extracellular matrix produced by the CSSCs and corneal crystallins produced by ICECs. ${ }^{22}$.

Degradability of PEG-collagen hydrogels. Hydrogel degradability is an important parameter for corneal repair and regeneration. Ideally, the hydrogel should not degrade too slowly or quickly in order to provide for proper tissue ingrowth. Here, the degradability of PEG-collagen hydrogels was evaluated after swelling in the presence of $1 \mathrm{mg} / \mathrm{mL}$ of collagenase and was monitored as a function of incubation time in complete supplemented Keratinocyte Serum Free (KSFM) medium at $37^{\circ} \mathrm{C}$ (Fig. 4a,b). In the presence of collagenase, noncrosslinked collagen hydrogels degraded to about $50 \%$ of its initial mass after $8 \mathrm{~h}$. After $12 \mathrm{~h}$, about $20 \%$ of the hydrogel mass remained. PEG-collagen hydrogel degradation was not dependent on the number of PEG arms and concentration at $12 \mathrm{~h}$. After $4 \mathrm{~h}$ in collagenase, the hydrogel mass was similar to time 0 for both 4 -arm PEG and 8-arm PEG-collagen hydrogels. After 8 h, 20\% of the PEG-collagen hydrogels was degraded, and after $12 \mathrm{~h}$, $30 \%$ to $40 \%$ of the hydrogels were degraded. Overall, the mass of the hydrogels decreased with the time in collagenase. The degradation of PEG-collagen hydrogels was statistically slower compared to non-crosslinked collagen hydrogels at all evaluated times $(p<0.05)$. Of note, we chose a collagenase concentration of $1 \mathrm{mg} / \mathrm{ml}$ since it provided more than $50 \%$ degradation of the non-crosslinked collagen hydrogel. In addition, we have used the same enzyme concentration that have been used in a previous publication to degrade corneal tissue ${ }^{23}$. In the presence of CSSCs, PEG-collagen hydrogel degradation occurred much slower compared to the hydrogels in the presence of collagenase (Fig. 4c). At 12 h, nearly $100 \%$ of the hydrogel initial weight was still present. However, non-crosslinked collagen hydrogel degradation in the presence of cells followed a similar profile to those in the presence of collagenase. To evaluate protein release from these hydrogels, human epithelial growth factor (EGF) was entrapped in the PEG-collagen hydrogels and EGF release was evaluated over one week without collagenase. The release of EGF from the hydrogels without collagenase incubation was slow and did not significantly vary with the type and PEG concentration (Fig. 4d). After 7 days, about 95\% of the EGF was still encapsulated in the PEG-collagen hydrogels.

ICEC growth on PEG-collagen hydrogels. We next evaluated PEG-collagen hydrogels' biocompatibility and their ability to support adhesion and proliferation of ICECs. ICEC adhesion on the hydrogels was evaluated $3 \mathrm{~h}$ after seeding the cells (Supplementary Fig. 1). We considered cell growth on the non-crosslinked (physically crosslinked) collagen hydrogel to be the point of comparison to calculate \% cell adhesion given that it is a widely used substrate for corneal epithelial cell growth in culture. ICEC adhesion did not significantly change 
a
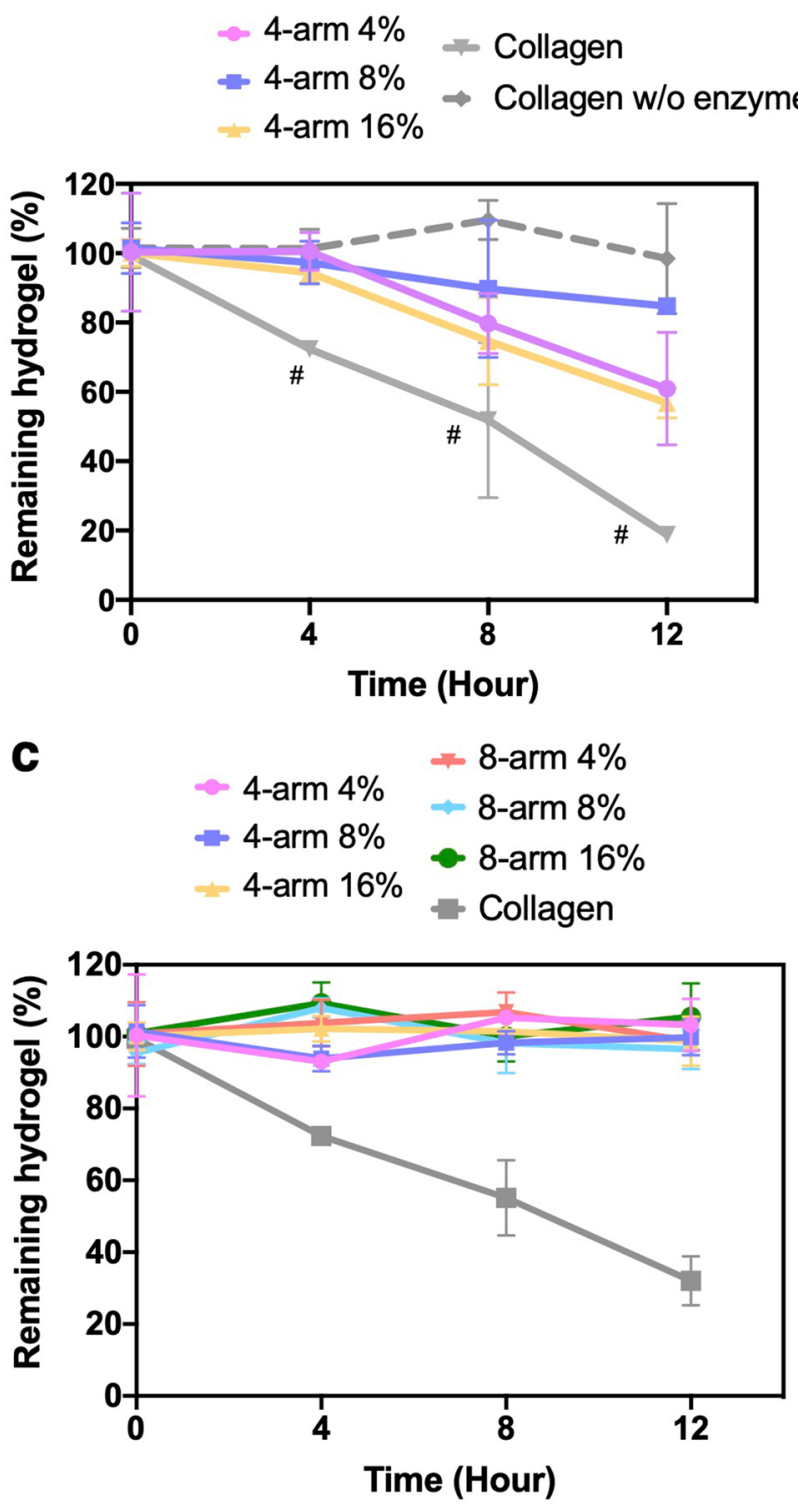

b
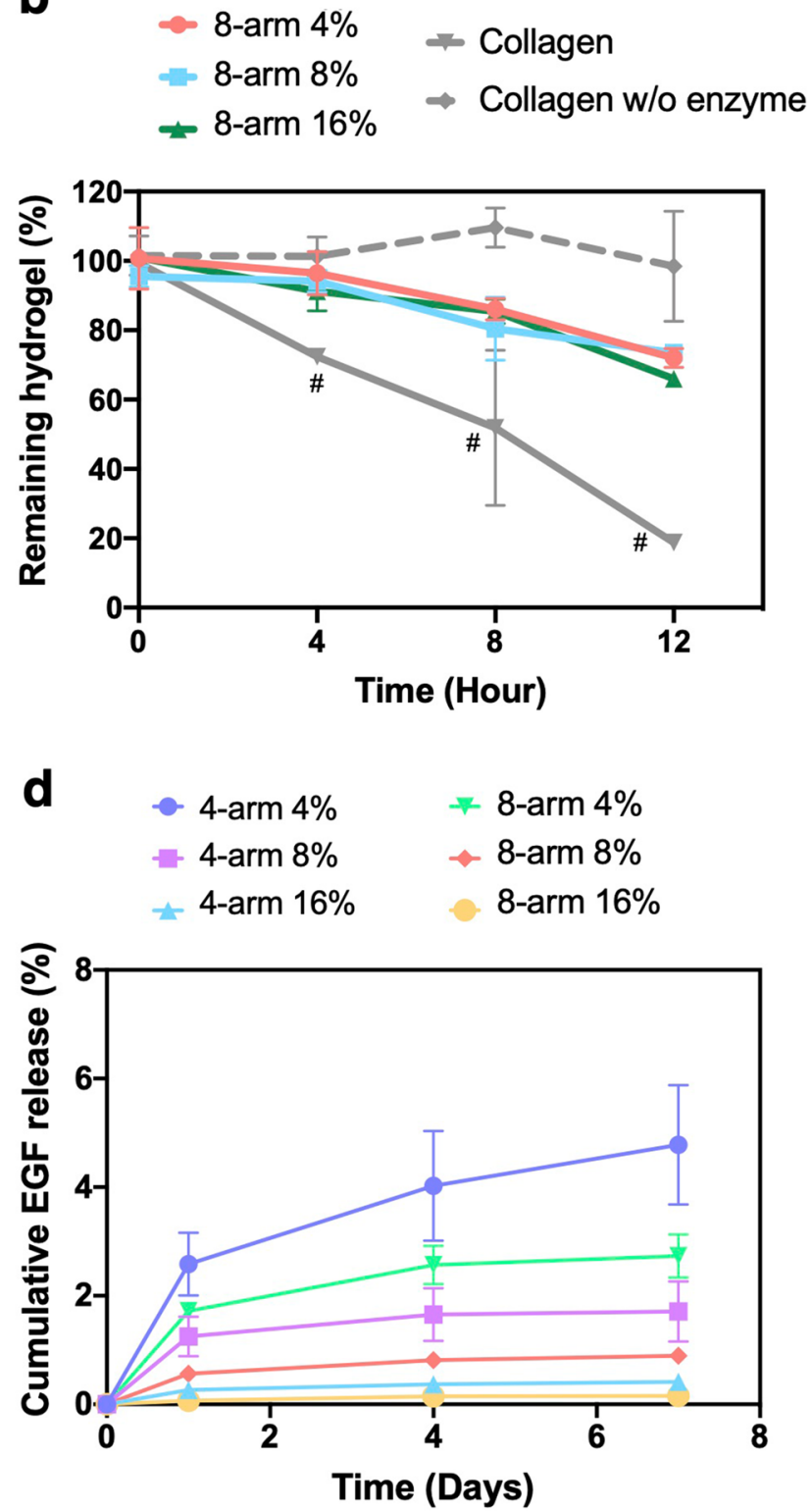

Figure 4. Degradation of (a) 4 and (b) 8-arm PEG-collagen hydrogels at different PEG concentrations (4\%, 8\%, and 16\%) $24 \mathrm{~h}$ after swelling was evaluated in the presence of $1 \mathrm{mg} / \mathrm{mL}$ of collagenase for $12 \mathrm{~h}$. Data is presented as mean $\pm \mathrm{SD}$, two-way ANOVA $(\mathrm{p}<0.005)$ was used to detect statistical differences followed by Tukey's multiple comparisons test. (c) Degradation profile of 4 and 8-arm PEG-collagen in the presence of CSSCs for $12 \mathrm{~h}$. (d) EGF release from 4 and 8-arm PEG-collagen hydrogels during 7 days in PBS. For each condition $\mathrm{n}=3$ was used.

between the two PEG arm numbers. ICEC adhesion on 4-arm PEG-collagen did not vary as a function of PEG concentration. The same was not observed for 8-arm PEG-collagen; ICECs exhibited better adhesion at higher PEG concentration (8 and 16\%) compared to 4\% PEG concentration $(p<0.05)$ (Supplementary Fig. 1a). Next we evaluated cell viability in the presence of the hydrogels. ICECs were seeded on the PEG-collagen hydrogels and incubated for 2 days in complete $\mathrm{KSFM}$ medium at $37^{\circ} \mathrm{C}$. Material biocompatibility was then evaluated using Live/Dead assay. 4-arm and 8-arm PEG-collagen hydrogel at varying concentrations showed low or no cytotoxicity as observed by the green staining (Live cells) and very few dead cells (red staining, arrows) (Fig. 5a). Quantification of live cells showed that nearly $100 \%$ of the cells were alive after 2 days in the presence of the hydrogels (Fig. 5b). Next, cell morphology was evaluated by staining with F-actin (Fig. 5c). ICEC growth on non-crosslinked collagen hydrogels was characterized by the presence of lamellipodia and few confluent areas. ICEC behavior did not vary with PEG arm number but was observed to vary with PEG concentration. ICECs that proliferated on PEG-collagen hydrogels at 4 and 8\% PEG concentration had significantly larger cell area compared to the cells that proliferated on non-crosslinked collagen hydrogels and on tissue culture polystyrene (TCP) plates ( $2 \mathrm{D}$ condition) at 2 days in culture, $p<0.05$ (Fig. $5 \mathrm{~d}$ ). This behavior was also observed for the 8 -arm PEG-collagen hydrogels. ICEC proliferation on PEG-collagen hydrogels was evaluated using MTT (Supplemen- 

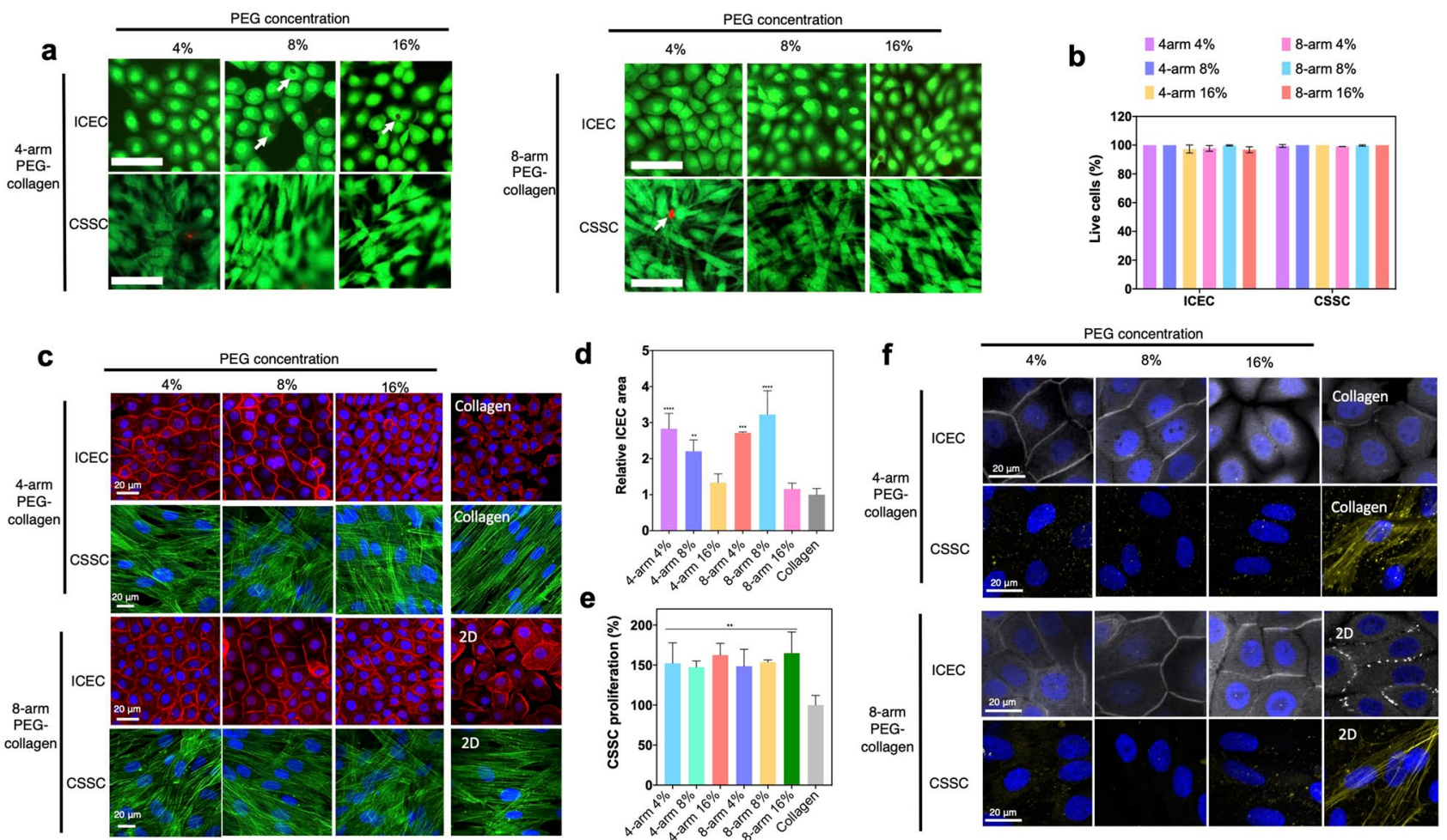

Figure 5. (a) Live/dead assays on ICEC and CSSC seeded on 4-arm PEG-collagen and 8-arm PEG-collagen hydrogels using different concentrations of PEG after 2 days in culture. Scale bar: $100 \mu \mathrm{m}$-live cells (green) and dead cells (red). (b) Viability of corneal cells seeded on PEG-collagen hydrogels. (c) F-actin staining ICEC (red) and CSSC (green) showing cell morphology when seeded on 4-arm PEG-collagen and 8-arm PEG-collagen hydrogels using different concentrations of PEG after 2 days in culture. The nucleus (blue) was stained for both cells. Scale bar: $20 \mu \mathrm{m}$. (d) Relative ICEC cell area 2 days after seeding on PEG-collagen hydrogel. Areas of the cells seeded on 4 and 8-arm PEG-collagen with 4 and 8\% PEG were statistically different compared to non-crosslinked collagen $\left({ }^{* * *} \mathrm{p}<0.0001,{ }^{* * *} \mathrm{p}=0.0002,{ }^{* *} \mathrm{p}=0.003\right)$. The data was normalized by cells seeded on non-crosslinked collagen hydrogels, with $\mathrm{n}=3$ for each condition and ordinary one-way ANOVA applied, followed by Dunnett's multiple comparison test. (e) Relative CSSC proliferation on 4-arm and 8-arm PEG-collagen hydrogels using different concentrations of PEG polymer after 2 days in culture. The data was normalized by cells seeded on non-crosslinked collagen hydrogels. A sample size of 3 and an ordinary one-way ANOVA was used followed by Dunnett's multiple comparison test. $\left({ }^{* *} \mathrm{p}<0.01 v s\right.$ non crosslinked collagen). (f) ZO-1 (gray) staining of ICEC and ASMA staining of CSSC proliferated on PEG-collagen hydrogels for 2 days. Scale bar: $20 \mu \mathrm{m}$.

tary Fig. 2). ICEC grown on the non-crosslinked collagen hydrogel was considered to be $100 \%$ proliferated. ICEC seeded on 4-arm PEG-collagen hydrogels at 4 and $8 \%$ PEG concentration proliferated significantly less than they did on 8-arm PEG-collagen for both 4 and 8\% of PEG concentrations, at the same time point (2 days). Interestingly, this behavior was not observed for hydrogels with 16\% PEG content. Overall, cell proliferation was greater on PEG-collagen hydrogels than it was on non-crosslinked collagen hydrogels. ICEC phenotype was evaluated for the cells grown on PEG-collagen hydrogels, non-crosslinked collagen and TCP by the expression of zonula occludens (ZO-1). ICEC that proliferated on the different scaffolds expressed ZO-1 suggesting that the cells form tight junctions characteristic of a normal epithelium (Fig. 5f). Overall, the expression of ZO-1 was greater for the cells that proliferated on both 4 and 8-arm PEG-collagen hydrogels at 4 and 8\% PEG content compared to the other conditions.

CSSC growth on PEG-collagen hydrogels. CSSC behavior on PEG-collagen hydrogels was also evaluated. CSSCs seeded on 4\% 4-arm PEG showed improved adhesion compared to cells seeded on 8-arm PEG hydrogel at the same concentration $\left(^{\# \#} p=0.005\right)$. For 4 arm PEG-collagen hydrogels, CSSC adhesion increased with the PEG concentration. CSSC seeded on 4 arm PEG-collagen with $16 \%$ PEG showed better adhesion compared to those with $4 \%$ PEG $\left({ }^{*} p=0.01\right)$. CSSC adhesion on 8 arm PEG-collagen hydrogel with 4 and $16 \%$ PEG content was significantly better compared to the cells seeded on $8 \%\left({ }^{\star *} p=0.006\right.$ and $\left.{ }^{\star} p=0.01\right)$ (Supplementary Fig. 1b). CSSC seeded on non-crosslinked collagen hydrogels expressed stress actin filaments that were primarily oriented in one direction, but the same was not observed for the CSSCs seeded on the PEG-collagen hydrogels (Fig. 5c). CSSC proliferation on PEG-collagen hydrogels was evaluated using MTT (Fig. 5e). CSSC grown on the non-crosslinked collagen hydrogel was considered as the baseline control (100\% proliferated). CSSC seeded on PEG-collagen hydrogels proliferated similarly at all concentrations and number of PEG arms. CSSCs seeded 
a

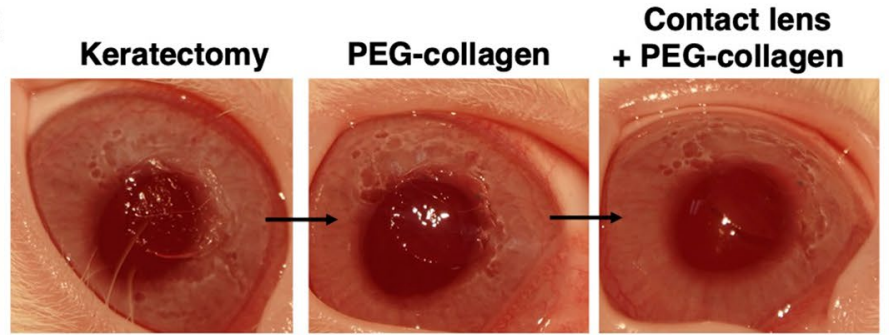

b

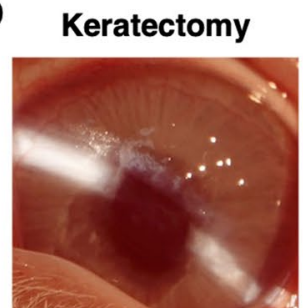

PEG-collagen

C
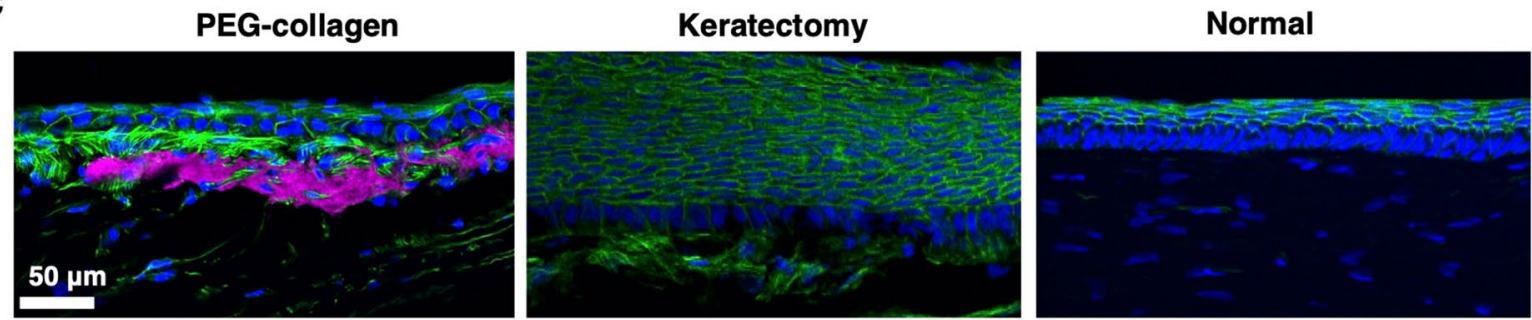

F-Actin

ASMA
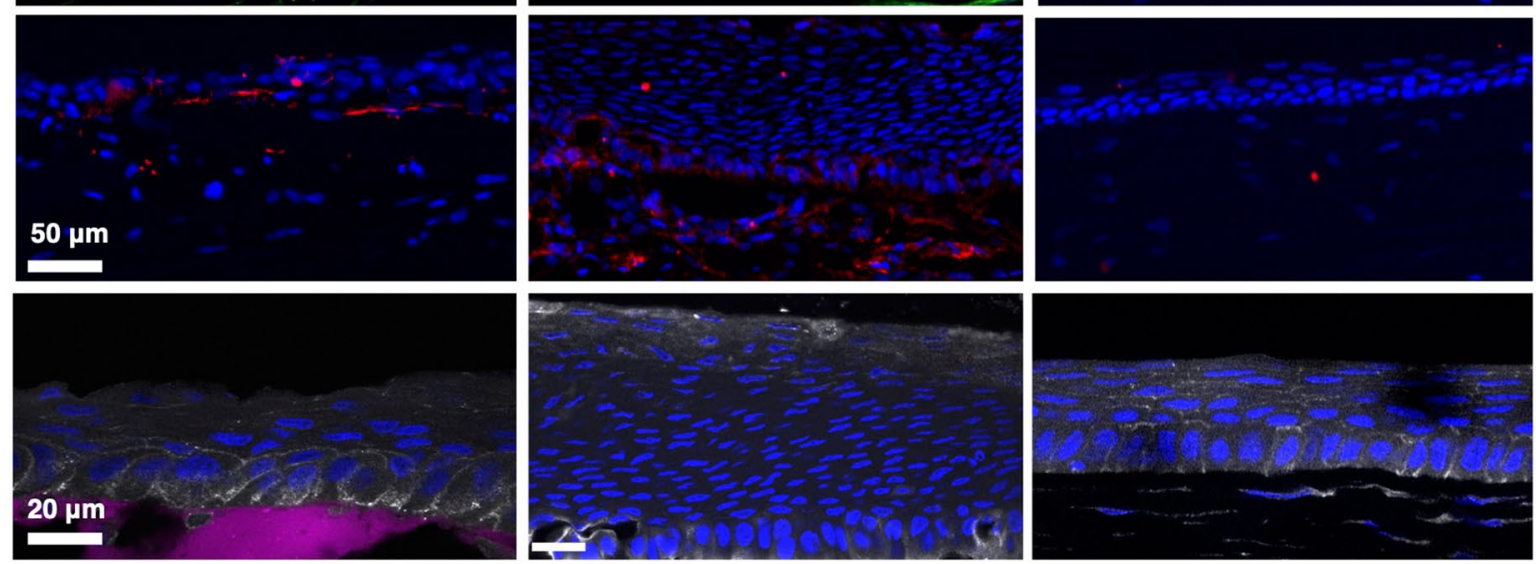

ZO-1

CK3
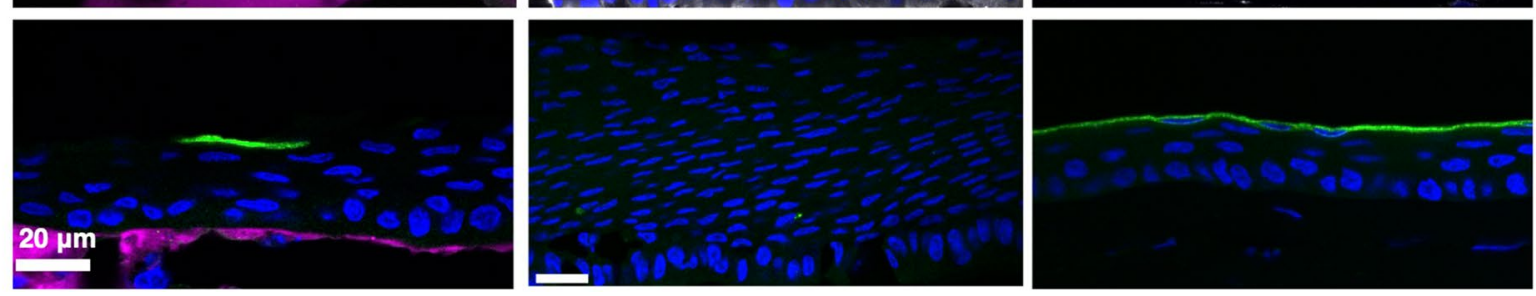

Figure 6. (a) Photographs of rabbit corneas right after keratectomy showing a rough surface and then a smooth surface after application of PEG-collagen hydrogel and contact lens. (b) Appearance of untreated and treated corneas on post-op day 7. (c) PEG-collagen hydrogel (stained magenta) underneath a multi-layered, migrated epithelial layer can be observed 7 days after treatment. For the keratectomy-only group, epithelial hyperplasia was observed. ASMA (red) was observed for the rabbits that received PEG-collagen as well as in the keratectomy group. Normal epithelial cell phenotype was detected by the presence of ZO-1 (gray) in the wing and superficial cells and CK3 (green). Minimal ZO-1 expression and no focal CK3 expression was observed for the keratectomy group.

on non-crosslinked collagen hydrogels proliferated to a lesser degree compared to PEG-collagen hydrogels. The transformation of CSSCs into myofibroblasts was evaluated after proliferation on the different hydrogels. We evaluated the expression of alpha smooth muscle actin (ASMA), a marker of myofibroblastic activity, generally present in scar tissue or fibrosis. We observed that some cells that proliferated on non-crosslinked collagen hydrogels and TCP exhibited ASMA expression (Fig. 5f). We did not observe ASMA expression in cells that proliferated on PEG-collagen hydrogels suggesting that these scaffolds are able to preserve a more quiescent, keratocytic phenotype.

In vivo evaluation of PEG-collagen hydrogels as fillers of corneal defects. Scaffold biointegration with host tissue is a requirement for proper tissue regeneration. Here we evaluated biointegration by the ability of the hydrogel to subsist on the stromal layer, after seven days in vivo, as well as by the phenotypic response of epithelial and stromal cells of the treated corneal tissue. Manual keratectomies were performed on the corneas of New Zealand white rabbits to simulate a stromal defect (Fig 6a, left photo). After the keratectomies were 
performed, the 4-arm PEG-collagen hydrogels with 8\% PEG content were applied to the defect sites (Fig 6a, middle photo) and then bandage contact lenses were placed on the eyes to protect the gel-treated cornea, followed by tarsorraphy (sutured eyelid closure). After 1 week, PEG-collagen hydrogels were able to support the growth and migration of epithelial and stromal cells (Fig. $6 c$, first column). Three rabbits were tested $(n=3)$. We selected the 4-arm PEG-collagen with $8 \%$ PEG on the basis of the fact that it exhibited the greatest transparency (Fig. 3) in the presence and absence of cells compared to all other formulations, in the context of the fact that it also compared well to other formulations in all other biophysical experiments. The impact of using the 8-arm PEG-collagen as an ocular adhesive to sustain cell migration and wound healing was also studied in one rabbit with similar results shown in Supplementary Fig. 4. Figure 6a shows that after the application of the hydrogel, the stromal defect becomes smooth compared to the rough surface right after keratectomy. Slit lamp exam on post-operative day 7 revealed that there were no clinical signs of anterior segment inflammation after treatment (Fig. 6b). One week after surgery, the animals were euthanized and the corneas were analyzed for the migration and proliferation of epithelial cells over the hydrogels, presence of ASMA, ZO-1 and CK3 (Fig. 6c). We found that the PEG-collagen hydrogels were able to support the growth and migration of epithelial cells and showed good apposition to the underlying stromal bed (Fig. 6c). Numerous epithelial layers strongly marked by F-actin staining were observed in the no treatment group indicative of epithelial hyperplasia (Fig. 6c). This was not observed in the groups that received the hydrogel treatment. Myofibroblasts were found to be present in both the treated and control corneas 7 days after the keratectomy. ZO-1 staining was observed in the epithelial superficial layer and wing cells of the treatment group after keratectomy (Fig. 6c). The control group showed ZO-1 staining in the most-superficial cells but not in the underlying wing cells. Epithelial cells that migrated over the hydrogel were able to express CK3 in some areas showing that the cells are differentiating into mature corneal epithelial cells. Corneas from the control group exhibited no focal expression of CK3, suggesting abnormal phenotype. In addition, 8-arm PEG-collagen hydrogel (8\%) was also able to support epithelial cell migration and phenotype while few ASMA positive cells were observed (Supplementary Fig. 4). In summary, PEG-collagen hydrogels were able to fill the defect area and remained transparent over one week and supported multi-layered epithelial growth along with markers of normal differentiation (ZO-1 and CK3).

\section{Discussion}

PEG-based ocular adhesives have been approved by the FDA to seal clear corneal incisions commonly used in cataract surgery ${ }^{24}$. In addition to PEG-based materials, currently available corneal adhesives are based on biological fibrin or synthetic cyanoacrylate glues, although both of these are used off-label. The main goal of cyanoacrylate, for instance, is to prevent corneal perforation and to serve as a temporizing measure before a corneal transplant ${ }^{25}$. Despite their nontoxicity and low immunogenicity, these material have some limitations with regard to stability and gelation time $e^{4}$ and, in the case of cyanoacrylate, a complete lack of optical transparency or biointegration. Alternative treatments for stromal defects are being explored that improve patient outcomessince there are no technologies specifically approved for the filling and regeneration of stromal defects. Collagen, hyaluronic acid and gelatin based fillers are being investigated as alternatives to the commercially available treatments to provide better biointegration with host tissue ${ }^{3,8,26}$. These corneal fillers should be degradable and support corneal cell migration and proliferation.

In prior work, we developed in situ-forming collagen hydrogels crosslinked by strain-promoted azide-alkyne cycloaddition (SPAAC) using bovine collagen type 1. These hydrogels were able to sustain the growth of both human keratinocytes and keratocytes ${ }^{12}$. Here we have developed in situ forming collagen hydrogels crosslinked by multi-functional PEG-N-hydroxysuccinimide that exhibited suitable mechanical and biological properties to fill and repair corneal stromal defects. PEG-collagen hydrogels have been previously evaluated for cytocompatibility, and fibroblast proliferation and migration ${ }^{17}$. In our system, bovine collagen type $1(3 \mathrm{mg} / \mathrm{mL}) \mathrm{was}$ crosslinked with multi-arm PEG-NHS via primary amine ester reaction. These hydrogels are formed in situ and are transparent and biocompatible. Our results showed that these hydrogels' mechanical properties can be tuned by varying PEG concentration and arm number. In addition, PEG concentration and arm number had effects on transparency, storage modulus and degradation profile. High concentration of PEG negatively impacted the gels' biophysical properties. For instance, the transparency and the storage modulus decreased at $16 \%$ PEG content. We believe that there was a saturation effect, where beyond a certain concentration of PEG, no further crosslinking was achieved, and any additional PEG served only to reduce crosslinking density and also decreased transparency via inadequate macromolecular mixing leading to matrix heterogeneity. At lower concentrations, crosslinking and mixing are well-balanced, leading to more transparent hydrogels.

Both PEG and collagen have been explored in tissue engineering due to their biocompatibility and ability to be easily modified to create in situ scaffolds with different mechanical properties ${ }^{17,27}$. These properties are particularly attractive for corneal regeneration applications that require safe and transparent scaffolds ${ }^{28}$. We showed that corneal epithelial and stromal cells were able to proliferate, adhere and had desirable morphology when grown on the PEG-collagen scaffolds. ICECs proliferate to a greater degree on PEG-collagen hydrogels versus non-crosslinked collagen and were able to form a monolayer. CSSCs did not vary between the PEG-collagen hydrogels in terms of morphology but were able to proliferate more effectively on crosslinked collagen hydrogels than on non-crosslinked collagen. The number of PEG arms but not the storage modulus impacted corneal cell behavior. The different behaviors observed for corneal cells on crosslinked and non-crosslinked collagen was previously investigated to be related to the alignment of the collagen fibers as a result of PEG crosslinking ${ }^{29}$. Here we chose to move forward with the 4 -arm PEG-collagen formulation with $8 \%$ PEG for the in vivo studies due to its superior transparency in the presence or absence of cells (Fig. 3) and the blend of desirable mechanical properties and cytocompatibility found through our in vitro experiments. The 8 -arm PEG-collagen at $8 \%$ 
PEG content did show promising wound healing results similar to those of the 4 -arm gel, but requires further evaluation with additional animals in future studies.

In vivo experiments revealed that the in situ forming PEG-collagen hydrogel was effective as a corneal defect filler that supported re-epithelialization in vivo one week after application. These hydrogels were likely bound to the stromal wound bed to some extent due to reactivity of NHS moieties with primary amines in stromal collagen ${ }^{17}$ given that the same chemistry is used to seal corneal incisions ${ }^{21}$. Of note, epithelial hyperplasia was observed in the keratectomy-only group and not the PEG-collagen treatment group, a phenomenon that is a function of stromal defect depth ${ }^{30}$. The presence of PEG-collagen hydrogels provided an exogenous stromal matrix on the wound bed that supported multi-layered epithelial migration and proliferation as well as ZO-1 and CK3 expression $^{31}$. In normal corneal stromal wound healing, corneal fibroblast infiltration followed by conversion to myofibroblasts is observed ${ }^{32}$. In our study, myofibroblastic responses were present in the cornea of animals that received the PEG-collagen gel and in the untreated corneas as expected after stromal injury, although to a qualitatively lesser extent in the treated corneas.

In summary, we have shown that collagen hydrogels crosslinked via multi-arm PEG succinimidyl esters can be formed rapidly in situ and can support multi-layered re-epithelialization when applied to corneal stromal defects. The hydrogels form rapidly under ambient conditions without the need for a chemical or photochemical trigger. The material represents a promising in situ stabilizer of corneal defects that, in contrast to cyanoacrylate glue, has the potential to maintain vision due to its transparency and promote healing by supporting surface epithelialization. Although they were found to degrade in the presence of collagenase in vitro, limited degradation was seen in vivo but is certain to occur over time, and thus longer follow up intervals are needed to understand what affect this has on the treated corneas in terms of transparency and anatomic outcomes. Additional studies that evaluate longer follow up periods, different wound diameters and depths, and additional formulation variations are merited to further understand the therapeutic potential of this material in the repair of corneal wounds.

\section{Methods}

Fabrication of PEG-collagen and non-crosslinked collagen hydrogels. Type I bovine collagen (Thermo Fisher Scientific; A1064401) was first $\mathrm{pH}$ neutralized using a solution of $1.0 \mathrm{M}$ sodium hydroxide solution, deionized (DI) water, and $10 \times \mathrm{PBS}$ in a 3:57:20 ratio. The $5 \mathrm{mg} / \mathrm{mL}$ collagen solution was mixed with the neutralization solution in 3:2 ratio so that the final concentration of collagen was $3 \mathrm{mg} / \mathrm{mL}$. Neutralized collagen was conjugated to 4 or 8 arm PEG Succinimidyl NHS ester $10 \mathrm{~K}$ (Creative PEGworks; PSB-4413 and PSB-846) via NHS chemistry to react with collagen's primary amines. First, PEG was solubilized in PBS to give a concentration of $100 \mathrm{mg} / \mathrm{mL}$, then 4,8 and $16 \mu \mathrm{L}$ of this solution was added to $100 \mu \mathrm{L}$ of neutralized collagen. Next, the mixed solution of PEG and collagen were put at $37^{\circ} \mathrm{C}$ for $30 \mathrm{~min}$ to ensure gelation of the hydrogels. For non-crosslinked collagen hydrogels, the collagen was neutralized as we mentioned above and incubated for $30 \mathrm{~min}$ at $37^{\circ} \mathrm{C}$ according to the collagen gelation procedure of Thermo Fisher Scientific. The non-crosslinked collagen is a physical crosslinked hydrogel formed by the neutralization of collagen at $37{ }^{\circ} \mathrm{C}$. Although it is not a covalently crosslinked system, it does form a physically crosslinked gel that is relatively strong and allow cell studies in these systems over several days ${ }^{12,33}$. To visualize the hydrogel for the in vivo experiments, Alexa Fluor 647 NHS Ester (Thermo Fisher Scientific, A20006) was conjugated to the neutralized collagen following the manufacturer's instructions. Briefly, the collagen conjugated to Alexa 647 was incubated for $2 \mathrm{~h}$ at $4{ }^{\circ} \mathrm{C}$ and dialyzed via Slide-A-Lyzer dialysis kit (Thermo Fisher Scientific, 66,382) overnight at $4{ }^{\circ} \mathrm{C}$ in PBS. Conjugated collagen $647(50 \mu \mathrm{L})$ was mixed with neutralized collagen $(100 \mu \mathrm{L})$ before adding the PEG-NHS reagent. Aseptic procedures were used at all times. For the in vitro and in vivo experiments, a bottle of sterile collagen type 1 bovine was opened under the hood where neutralized collagen and PEG was mixed together to form hydrogels as described above. For the in vivo studies, the PEG and collagen reagents were prepared under the hood and only opened at the time of surgery on a sterile field and handled using sterile instruments.

Mechanical characterization of PEG-collagen hydrogel. The mechanical properties of the collagen hydrogels were evaluated using an ARES-G2 rheometer (TA Instruments, New Castle, DE, USA) at Stanford Soft \& Hybrid Materials Facility (SMF, Stanford, CA, USA). For the non-crosslinked collagen hydrogel, neutralized collagen was mounted on the plate and measured. For the PEG-collagen hydrogels 4 or 8-arm PEG NHS solution was added to the neutralized collagen solution. Then the $150 \mu \mathrm{l}$ of mixed solution was mounted on the plate immediately after mixing with a pipette. To determine gelation time, time sweeps were performed at $37^{\circ} \mathrm{C}$ for $1200 \mathrm{~s}$ at $1 \%$ strain and $1 \mathrm{~Hz}$ oscillatory frequency. Next, frequency sweeps from 0.1 to $10 \mathrm{~Hz}$ with a fixed $1 \%$ strain were performed to determine the completion of gelation. To evaluate how the mechanical properties of the hydrogels could be modulated, PEG-collagen hydrogels were formed at different concentrations 4, 8 and $16 \%$ of PEG to collagen. To examine the mechanical properties at complete gelation, the resultant solutions were deposited onto a glass substrate and incubated at $37^{\circ} \mathrm{C}$ for $2 \mathrm{~h}$, and then frequency sweeps from 0.1 to $10 \mathrm{~Hz}$ with a fixed $1 \%$ strain were performed. The storage moduli was found to nearly equivalent to those found at $1200 \mathrm{~s}$ in Fig. 2a.

PEG-collagen hydrogel transparency evaluation. The hydrogels' absorbance from 350 to $800 \mathrm{~nm}$ was measured using a SpectraMax M Series Multi-Mode Microplate Reader before and $24 \mathrm{~h}$ after swelling in 100 $\mu \mathrm{L}$ of PBS. The PEG-collagen and non-crosslinked collagen hydrogels were fabricated in a 96 well plate, and the volume was $100 \mu \mathrm{L}$. The hydrogel transmittance was also evaluated at $24 \mathrm{~h}$ in the presence of the cornea cells. Briefly, $1 \times 10^{5}$ cells $/ \mathrm{mL}$ were seeded on the hydrogels in a 96 well plate and $24 \mathrm{~h}$ later, the medium was removed and loose cells were washed out with PBS before reding the hydrogel's absorbance. The absorbance was converted to transmittance using the relation $\mathrm{A}=2-\log 10(\% \mathrm{~T})$. For each condition, $\mathrm{n}=3$ was used and the 
absorbance of the medium without phenol red was subtracted from the hydrogel's absorbance before converting to transmittance.

Multi-arm PEG-collagen hydrogel degradation. We used two methods for evaluating the degradation of 4 and 8-arm PEG-collagen hydrogels with 4\%, 8\%, and 16\% PEG content and non-crosslinked collagen hydrogels. Degradation was studied in the presence of collagenase or in the presence of seeded CSSC cells. Eppendorf tubes were weighed and $70 \mu \mathrm{L}$ of each hydrogel was synthesized inside of the tubes. Next, $140 \mu \mathrm{L}$ of $1 \mathrm{mg} / \mathrm{mL}$ of Collagenase from Clostridium histolyticum type I, 0.25-1.0 FALGPA units/mg solid, $\geq 125$ CDU/ mg (Sigma Life Sciences; C0130) in warm supplemented Keratinocyte Serum Free Medium-KSFM (Thermo Fisher Scientific; 17,005,042) containing Bovine Pituitary Extract (BPE), EGF, $100 \mathrm{ng} / \mathrm{mL}$ of hydrocortisone (Sigma Aldrich; H0008) and $5 \mu \mathrm{g} / \mathrm{mL}$ of insulin (Sigma Aldrich; 0516) was added to the tubes containing the hydrogel. Untreated hydrogels received $140 \mu \mathrm{L}$ of warm KSFM with no collagenase. The tubes were placed on a platform rocker, allowing the collagenase to react with the hydrogel. For hydrogel degradation in the presence of seeded CSSC cells, $1 \times 10^{5}$ cells $/ \mathrm{mL}$ were added to the swollen hydrogels in cell culture media. After 0 , 4,8 , and $12 \mathrm{~h}$, the supernatant was removed, leaving the remaining hydrogel behind (with or without cells). At each time the tubes were weighed and subtracted from the original weight of the tube with hydrogel (time 0 ) to obtain the wet weight of the hydrogel. For each condition $n=3$ was used. The hydrogel weight at time 0 was considered $100 \%$ of the hydrogel weight. The degradation of the hydrogels was compared to the degradation of non-crosslinked collagen hydrogel with and without collagenase or cells (control).

Epidermal growth factor release. The EGF release was evaluated as previously described ${ }^{34,35}$. Briefly, the PEG-collagen hydrogel including EGF was fabricated in 48-well plates. The initial volume of solution was $100 \mu \mathrm{l}$. After mixing neutralized collagen with 4 or 8 arm PEG-NHS solution in each well, the hydrogel was incubated at $37{ }^{\circ} \mathrm{C}$ for $30 \mathrm{~min}$. At each well, $500 \mu \mathrm{l}$ of PBS solution was applied, and the solution was collected and refreshed at each time point. The amount of released EGF was measured by EGF ELISA kit (30X wash buffer, biotinylated antibody reagent, streptavidin-HRP concentrate, TMB substrate, and stop solution containing $0.16 \mathrm{M}$ sulfuric acid; Thermo Fisher Scientific, Waltham, MA, USA), and we followed the EGF ELISA kit protocol. Briefly, the collected solutions were added to the capture antibody-coated well plate and incubated at $37{ }^{\circ} \mathrm{C}$ for $2 \mathrm{~h}$. The biotinylated antibody reagent and diluted streptavidin-HRP in PBS were added to the well and incubated for 2 and $1 \mathrm{~h}$ in turn, respectively. After each step, all wells were washed three times using the washing buffer in the kit. For the color development, TMB solution was added and incubated in the dark for 30 min at room temperature, and stop solution was added to stop the reaction without washing. The absorbance of each resultant well was measured at $550 \mathrm{~nm}$ using SpectraMax M Series Multi-Mode Microplate Reader (Sunnyvale, CA, USA). For quantification of immobilized EGF, a standard curve of EGF ELISA was obtained following the established protocol of instruction, and the amount of EGF was calculated. The total amount of added EGF was calculated, and the released amount of EGF was divided by the total amount of initial EGF.

Corneal epithelial cell culture. ICEC were kindly donated by Dr. Djalilian's laboratory from the University of Chicago, Illinois. ICECs were culture in supplemented KSFM. After the cells reached 80\% confluency, they were passaged. The medium was changed every other day.

Corneal stromal stem cell culture. CSSC were harvested from human donor corneas provided by Lions Eye Institute. Research corneas were in Optisol GS had minimum cell count of 2000, maximum death to preservation time of 7 days, and no history of HSV/VZV/HIV/Hepatitis, age ranging from 30-35 years old. First, the endothelial layer was removed, as well as the central corneal using a trephine $(8 \mathrm{~mm})$. The remaining limbal region was dissected into small fragments and placed epithelial side down on 6 well plates. CSSCs were given time to transplant onto a flask bottom over a period of 7 days in Minimum Essential Medium Eagle (Sigma Aldrich; M4526) containing 10\% Fetal Bovine Serum (FBS), $1 \%$ antibiotic antimycotic solution (Sigma Aldrich; A5955), 1\% Non-essential Amino Acid Solution (Sigma Aldrich; M7145) and 1\% Glutamax (Thermo Fisher Scientific; $35,050,061)$. After reaching $80 \%$ confluence, the CSSC culture was subcultured into colonies and used until passage 4.

Corneal cell biocompatibility, morphology and proliferation on multi-arm PEG-collagen hydrogels. Cornea cell viability was evaluated using Live/Dead assay according to Thermo Fisher Scientific protocol. Briefly, $200 \mu \mathrm{L}$ of PEG-collagen hydrogels were formed within a 48 well plate as previously mentioned. Complete gelation of the hydrogel was achieved by incubating the hydrogels at $37^{\circ} \mathrm{C}$ for 20 to 30 min.". Next, supplemented KSFM was added to the hydrogels and left overnight. Next, $2 \times 10^{5}$ cells were seeded on the hydrogels for two days. Live/dead solution was added, and the cells were evaluated under inverted microscopic. Cellular morphological behavior of ICEC and CSSC were evaluated by staining the cells with Alexa Fluor 488 Phalloidin (Thermo Fisher Scientific; A12379) and Rhodamine Phalloidin (Thermo Fisher Scientific; R415). Phenotype evaluation was assessed by staining the cells with ZO-1(Thermo Fisher Scientific; ZO1-1A12) and ASMA (Abcam; ab5694). First, the hydrogels $(130 \mu \mathrm{L})$ were formed in an 8 well chamber slide and $5 \times 10^{4}$ cells were seeded on the hydrogel. After 2 days, the cells were fixed with $4 \%$ paraformaldehyde (PFA) for 15 min, permeabilized and blocked with $0.5 \%$ triton-X and 5\% goat serum (GS) in PBS for $30 \mathrm{~min}$. Primary antibodies in permeabilizing and blocking solution were added and incubated overnight at $4{ }^{\circ} \mathrm{C}$. In the next day, secondary antibody Alexa Fluor 488 in the same antibody solution was added for 2 h. Then Alexa Fluor 488 Phalloidin or Rhodamine Phalloidin were added to the cells in PBS (1:40) for $30 \mathrm{~min}$. Next, 4',6-diamidino-2-phenylindole (DAPI) was added in PBS for $5 \mathrm{~min}$. The cells were then analyzed using confocal microscopy. Cell proliferation 
was evaluated using Thiazolyl Blue Tetrazolium Bromide (MTT, Sigma Aldrich; M5655). Briefly, the cells $5 \times 10^{4}$ were seeded on PEG-collagen hydrogels formed in 96 well plates. After 2 days, the MTT solution was added for $2 \mathrm{~h}$. The crystals were solubilized using Dimethyl Sulfoxide (DMSO). The absorbance was read at $570 \mathrm{~nm}$. Corneal cells plated on the wells without hydrogels, were considered to have $100 \%$ of proliferation.

In vivo keratectomy studies in rabbits. Adult New Zealand white rabbits were used to conduct lamellar keratectomy studies. Animal experiments were designed to conform with the ARVO statement for the Use of Animals in Ophthalmic and Vision Research and were reviewed and approved by the Stanford University Institutional Animal Care and Use Committee. All anesthesia techniques were performed by the veterinary service center (VSC) at Stanford University. Prior to surgery, one drop of proparacaine hydrochloride ophthalmic solution was added to the eye receiving treatment. A manual lamellar keratectomy was performed on the right eye using a $3.5 \mathrm{~mm}$ customized vacuum trephine to create a deep circular cut and a spatula was used to remove the anterior stroma. Approximately $5 \mu \mathrm{L}$ of 4 -arm PEG-collagen-Alexa 647 hydrogels at $8 \%$ PEG concentration were applied to the keratectomy area and allowed to gel in situ for $1 \mathrm{~min}$ before applying the contact lens and performing a tarsorrhaphy. The corneas from the 4 -arm PEG-collagen at $8 \%$ group $(n=3)$ were compared to the keratectomy group $(n=3$, control) clinically for signs of inflammation or infection. The 8 arm PEG-collagen was also applied to the keratectomy area of a rabbit cornea $(n=1)$. Ofloxacin ophthalmic solution was applied daily. On day 7, the tarsorrhaphy and the contact lens were removed for clinical examination under a surgical microscope and photographs using a Paxos Scope smartphone-based ophthalmic camera adapter. On day 7, the rabbits' eyes were enucleated for immunohistochemical processing and evaluation.

Immunohistochemistry. The tissues were sectioned, and cross-sections of corneal tissues were fixed with PFA 4\%, permeabilized and blocked as mentioned above. Primary antibodies ASMA, ZO-1 and CK-3 (Abcam; ab77869) were incubated in $0.5 \%$ triton-x and $5 \%$ NGS overnight. Next the secondary antibody anti-mouse Alexa 488 for CK3 was added for $2 \mathrm{~h}$. Finally, F-actin and DAPI were added for 30 and 5 min, respectively. The corneas cross-sections were analyzed under confocal microscopy.

Statistical analysis. All data are expressed as the mean \pm standard deviation (SD). Each experiment was repeated at least 3 times unless otherwise indicated. Statistical evaluation performed are informed on each Figure. A value of $p<0.05$ was considered statistically significant. The statistical analysis was performed by using GraphPad Prism 7 statistical software ${ }^{35}$.

\section{Data availability}

The analyzed datasets from this study are available from the corresponding author upon request.

Received: 3 March 2020; Accepted: 28 July 2020

Published online: 07 October 2020

\section{References}

1. Channa, R. et al. Epidemiology of eye-related emergency department visits. JAMA Ophthalmol. 134, 312-319. https://doi.org/10. 1001/jamaophthalmol.2015.5778 (2016).

2. Lagali, N. Corneal stromal regeneration: current status and future therapeutic potential. Curr. Eye Res. https://doi.org/10.1080/ 02713683.2019.1663874 (2019).

3. Samarawickrama, C. et al. Collagen-based fillers as alternatives to cyanoacrylate glue for the sealing of large corneal perforations. Cornea 37, 609-616. https://doi.org/10.1097/ico.0000000000001459 (2018).

4. Trujillo-de Santiago, G. et al. Ocular adhesives: design, chemistry, crosslinking mechanisms, and applications. Biomaterials 197, 345-367. https://doi.org/10.1016/j.biomaterials.2019.01.011 (2019).

5. Gain, P. et al. Global survey of corneal transplantation and eye banking. JAMA Ophthalmol. 134, 167-173. https://doi.org/10.1001/ jamaophthalmol.2015.4776 (2016)

6. Chen, Z. et al. Biomaterials for corneal bioengineering. Biomed. Mater. 13, 032002. https://doi.org/10.1088/1748-605X/aa92d2 (2018).

7. Kilic Bektas, C. \& Hasirci, V. Cell loaded 3D bioprinted GelMA hydrogels for corneal stroma engineering. Biomater. Sci. 8, $438-449$. https://doi.org/10.1039/C9BM01236B (2020).

8. Koivusalo, L. et al. Tissue adhesive hyaluronic acid hydrogels for sutureless stem cell delivery and regeneration of corneal epithelium and stroma. Biomaterials 225, 119516. https://doi.org/10.1016/j.biomaterials.2019.119516 (2019).

9. Kong, B. et al. Tissue-engineered cornea constructed with compressed collagen and laser-perforated electrospun mat. Sci. Rep. 7, 970. https://doi.org/10.1038/s41598-017-01072-0 (2017).

10. Fagerholm, P. et al. Stable corneal regeneration four years after implantation of a cell-free recombinant human collagen scaffold. Biomaterials 35, 2420-2427. https://doi.org/10.1016/j.biomaterials.2013.11.079 (2014).

11. Pellegrini, G. et al. Navigating market authorization: the path holoclar took to become the first stem cell product approved in the european union. Stem Cells Transl. Med. 7, 146-154. https://doi.org/10.1002/sctm.17-0003 (2018).

12. Lee, H. J., Fernandes-Cunha, G. M., Na, K.-S., Hull, S. M. \& Myung, D. Bio-orthogonally crosslinked, in situ forming corneal stromal tissue substitute. Adv. Healthc. Mater. 7, 1800560. https://doi.org/10.1002/adhm.201800560 (2018).

13. Li, L. et al. Gelatin-based photocurable hydrogels for corneal wound repair. ACS Appl. Mater. Interfaces 10, 13283-13292. https:// doi.org/10.1021/acsami.7b17054 (2018).

14. Palchesko, R. N., Carrasquilla, S. D. \& Feinberg, A. W. Natural biomaterials for corneal tissue engineering, repair, and regeneration. Adv. Healthc. Mater. 7, 1701434. https://doi.org/10.1002/adhm.201701434 (2018).

15. Yue, B. Biology of the extracellular matrix: an overview. J. Glaucoma 23, S20-S23. https://doi.org/10.1097/IJG.0000000000000108 (2014).

16. Latifi, N., Asgari, M., Vali, H. \& Mongeau, L. A tissue-mimetic nano-fibrillar hybrid injectable hydrogel for potential soft tissue engineering applications. Sci. Rep. 8, 1047. https://doi.org/10.1038/s41598-017-18523-3 (2018).

17. Sargeant, T. D., Desai, A. P., Banerjee, S., Agawu, A. \& Stopek, J. B. An in situ forming collagen-PEG hydrogel for tissue regeneration. Acta Biomater. 8, 124-132. https://doi.org/10.1016/j.actbio.2011.07.028 (2012). 
18. Assmann, A. et al. A highly adhesive and naturally derived sealant. Biomaterials 140, 115-127. https://doi.org/10.1016/j.bioma terials.2017.06.004 (2017).

19. Raiskup, F. \& Spoerl, E. Corneal crosslinking with riboflavin and ultraviolet A. I. Principles. Ocular Surf. 11, 65-74. https://doi. org/10.1016/j.jtos.2013.01.002 (2013).

20. Matossian, C., Makari, S. \& Potvin, R. Cataract surgery and methods of wound closure: a review. Clin. Ophthalmol 9, 921-928. https://doi.org/10.2147/OPTH.S83552 (2015).

21. Nallasamy, N., Grove, K. E., Legault, G. L., Daluvoy, M. B. \& Kim, T. Hydrogel ocular sealant for clear corneal incisions in cataract surgery. J. Cataract Refract. Surg. 43, 1010-1014. https://doi.org/10.1016/j.jcrs.2017.05.035 (2017).

22. Jester, J. V. et al. The cellular basis of corneal transparency: evidence for 'corneal crystallins' J. Cell Sci. 112, 613-622 (1999).

23. Choi, J. S. et al. Factors affecting successful isolation of human corneal endothelial cells for clinical use. Cell Transpl. 23, 845-854. https://doi.org/10.3727/096368913x664559 (2014).

24. Masket, S. et al. Hydrogel sealant versus sutures to prevent fluid egress after cataract surgery. J. Cataract Refract. Surg. 40, 20572066. https://doi.org/10.1016/j.jcrs.2014.03.034 (2014).

25. Yin, J. et al. Outcomes of cyanoacrylate tissue adhesive application in corneal thinning and perforation. Cornea 38, 668-673. https://doi.org/10.1097/ico.0000000000001919 (2019).

26. Shirzaei Sani, E. et al. Sutureless repair of corneal injuries using naturally derived bioadhesive hydrogels. Sci. Adv. 5, 1281-1281. https://doi.org/10.1126/sciadv.aav1281 (2019).

27. Rafat, M. et al. PEG-stabilized carbodiimide crosslinked collagen-chitosan hydrogels for corneal tissue engineering. Biomaterials 29, 3960-3972. https://doi.org/10.1016/j.biomaterials.2008.06.017 (2008).

28. Garagorri, N. et al. Keratocyte behavior in three-dimensional photopolymerizable poly(ethylene glycol) hydrogels. Acta Biomater. 4, 1139-1147. https://doi.org/10.1016/j.actbio.2008.05.007 (2008).

29. Lin, S. \& Gu, L. Influence of crosslink density and stiffness on mechanical properties of type I collagen gel. Materials (Basel) 8 , 551-560. https://doi.org/10.3390/ma8020551 (2015).

30. Eagle, R. C. Jr., Dillon, E. C. \& Laibson, P. R. Compensatory epithelial hyperplasia in human corneal disease. Trans. Am. Ophthalmol. Soc. 90, 265-276 (1992).

31. Suzuki, K., Tanaka, T., Enoki, M. \& Nishida, T. Coordinated reassembly of the basement membrane and junctional proteins during corneal epithelial wound healing. Invest. Ophthalmol. Vis. Sci. 41, 2495-2500 (2000).

32. Wilson, S. E. Corneal myofibroblast biology and pathobiology: generation, persistence, and transparency. Exp Eye Res. 99, 78-88. https://doi.org/10.1016/j.exer.2012.03.018 (2012).

33. Chuang, C. H., Lin, R. Z., Melero-Martin, J. M. \& Chen, Y. C. Comparison of covalently and physically cross-linked collagen hydrogels on mediating vascular network formation for engineering adipose tissue. Artif Cells Nanomed. Biotechnol. 46, S434-s447. https://doi.org/10.1080/21691401.2018.1499660 (2018).

34. Lee, H. J., Fernandes-Cunha, G. M., Putra, I., Koh, W.-G. \& Myung, D. Tethering growth factors to collagen surfaces using copperfree click chemistry: surface characterization and in vitro biological response. ACS Appl. Mater. Interfaces 9, 23389-23399. https:// doi.org/10.1021/acsami.7b05262 (2017).

35. Fernandes-Cunha, G. M. et al. Immobilization of growth factors to collagen surfaces using pulsed visible light. Biomacromol 18, 3185-3196. https://doi.org/10.1021/acs.biomac.7b00838 (2017).

\section{Acknowledgements}

This work was supported by the National Eye Institute (NIH K08 EY028176 and P30 EY026877), a core grant and Career Development Award from Research to Prevent Blindness (RPB), a grant from the Matilda Ziegler Foundation, the Stanford SPARK Program and Maternal \& Child Health Research Institute, the Veterans Affairs Small Projects in Rehabilitation Research (SPiRE) program (I21 RX003179), and the Byers Eye Institute at Stanford. The authors would like to thank Dr. Djalilian's lab for kindly providing the ICECs used in these studies. Part of this work was performed at the Stanford Nano Shared Facilities (SNSF), supported by the National Science Foundation under Award ECCS-1542152. The funders had no role in study design, data collection and analysis, decision to publish, or preparation of the manuscript.

\section{Author contributions}

(GM. F-C; K.C) contributed equally to this work; Design of the study (GM.F-C, K.C., D.M.); Conduct of study (GM.F-C., K.C., F.C, P.L, J.H. L.M, K.N., H.L.) Interpretation of the data (GM.F-C, K.C., H.L., D.M.); Preparation, review, or approval of the manuscript (GM.F-C, K.C., D.M.).

\section{Competing interests}

Author D.M. holds a patent on the portable ophthalmic camera system used to photograph the animal eyes, and authors D.M., GM.F-C, and H.L. have a patent application on the hydrogel technology used in the studies. All other authors declare no competing interests.

\section{Additional information}

Supplementary information is available for this paper at https://doi.org/10.1038/s41598-020-72978-5.

Correspondence and requests for materials should be addressed to D.M.

Reprints and permissions information is available at www.nature.com/reprints.

Publisher's note Springer Nature remains neutral with regard to jurisdictional claims in published maps and institutional affiliations. 
(c) (i) Open Access This article is licensed under a Creative Commons Attribution 4.0 International cc) License, which permits use, sharing, adaptation, distribution and reproduction in any medium or format, as long as you give appropriate credit to the original author(s) and the source, provide a link to the Creative Commons licence, and indicate if changes were made. The images or other third party material in this article are included in the article's Creative Commons licence, unless indicated otherwise in a credit line to the material. If material is not included in the article's Creative Commons licence and your intended use is not permitted by statutory regulation or exceeds the permitted use, you will need to obtain permission directly from the copyright holder. To view a copy of this licence, visit http://creativecommons.org/licenses/by/4.0/.

This is a U.S. Government work and not under copyright protection in the US; foreign copyright protection may apply 2020 